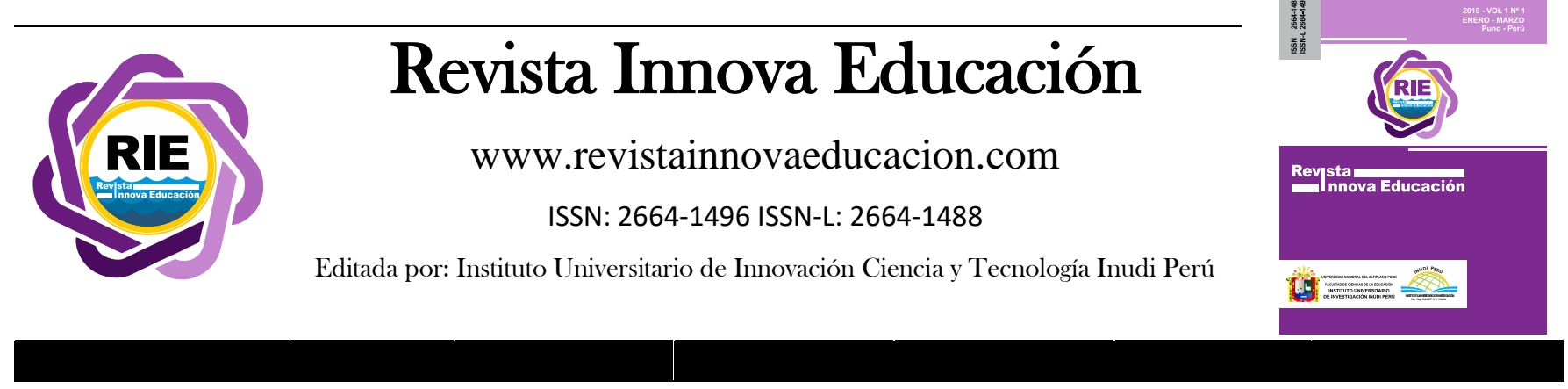

\title{
Influencia de la concepción cristiana en la actitud de liderazgo social de los estudiantes universitarios
}

\author{
Influence of the Christian conception on the attitude of social leadership of university students
}

\author{
William Mamani-Apaza (D); Pedro Huayanca (D); Marisol Yana
}

DOI: https://doi.org/10.35622/j.rie.2019.03.009

Universidad Nacional del Altiplano, Facultad de Ciencias de la Educación.

Av. Floral No 1153, Ciudad Universitaria, Puno, Perú.

Recibido el 03/06/2019/ Aceptado el 17/07/2019

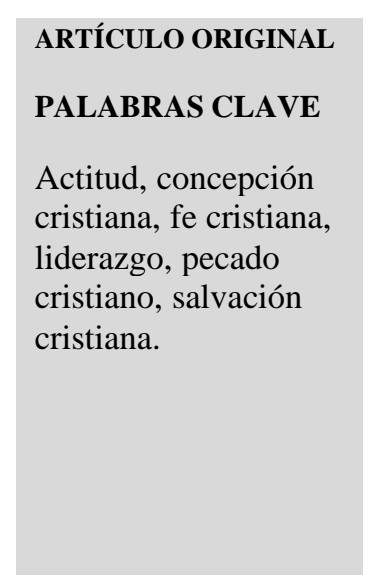

\section{KEYWORDS}

Attitude, Christian conception, Christian faith, leadership, Christian sin, Christian salvation.
El liderazgo social y la concepción cristiana son temas pocos tratados en el mundo académico. Por lo que el estudio sobre el pensamiento y liderazgo tiene como objetivo principal el de determinar la influencia de la concepción cristiana en la actitud de liderazgo social de los estudiantes. El tipo de investigación es no experimental descriptivo. Por la naturaleza de estudio se aplicó el diseño descriptivo explicativo. Asimismo, por la complejidad de la investigación, se aplicó el diseño analítico y causal. Como instrumento se utilizó una encuesta estructurada. Este estudio concluye que la concepción cristiana influye negativamente en la actitud de liderazgo social de los estudiantes, debido a que las manifestaciones conceptuales y prácticas en relación a la fe, salvación y el pecado buscan la pasividad, consolidando actitudes negativas para el desarrollo del liderazgo social, al mismo tiempo, sea útil a la sociedad. Esta aseveración queda confirmada mediante el diseño estadístico aplicado, Chi cuadrada, que fue contundente; es decir: $|164,6|>|8,0|$ de nivel de influencia.

Social leadership and Christian conception are few topics discussed in the academic world. So, the study on thinking and leadership has as its main objective to determine the influence of the Christian conception on the attitude of social leadership of the students. The type of research is non-experimental descriptive. Due to the nature of the study, the descriptive descriptive design was applied. Also, due to the complexity of the investigation, the analytical and causal design was applied. As a tool, a structured survey was used. This study concludes that the Christian conception negatively influences the attitude of social leadership of students, because the conceptual and practical manifestations in relation to faith, salvation and sin seek passivity, consolidating negative attitudes for the development of social leadership At the same time, be useful to society. This assertion is confirmed by the statistical design applied, Chi square, which was blunt; that is: $|164.6|>|8.0|$ of level of influence. 


\section{INTRODUCCIÓN}

El imperativo del estudio radica en proporcionar el marco teórico y metodológico para el análisis sistémico del liderazgo social. Asimismo, se pretende generar debate acerca de los distintos tipos de liderazgo, principalmente el del liderazgo social cognitivo, afectivo y conductual. Porque el Perú carece de líderes sociales comprometidos con la sociedad y con visión de desarrollo social. La dirección de instituciones educativas se ha reducido a administrar recursos materiales más que el recurso y el talento humano. Porque la potencia del liderazgo se enraíza cada vez más en las diferentes organizaciones, como escuelas, universidades, organismos sociales y empresariales, siendo lugares donde ejercen el nivel de mayor movilidad de los imaginarios sociales, y es aquí donde se construyen estrategias para crear hábitos y modificar conductas (Pautt, 2011).

La concepción pedagógica moderna ha reducido la función docente como líder. La esencia de la pedagogía sólo se ha conceptualizando en la transmisión de modelos euro-constructivistas. Sin considerar que el Perú es una realidad distinta. Estos modelos centristas han convertido al maestro estrictamente como "facilitadores" de los procesos pedagógicos como el del proceso de aprendizaje (Quiroz, 1998).

El enfoque constructivista reduce el liderazgo docente, su papel de líder; convirtiendo al maestro en meros mediadores y transmisor de conocimientos. Olvidando que, los valores son virtudes asociados a la firmeza de acción, a la eficacia de acción del ser humano y que se constituyen en referentes para guiar la conducta de los seres humanos (Terrazas, 2015). En este escenario un líder debe poseer valores inmanentes que permitan guiar al grupo hacia un derrotero final y beneficioso Dado que el constructivismo asume como principio la competencia, que requiere competentes, capaces de todo, cuyo centro es el aprendiz. Se debe tener cuidado, con esta afirmación, ya Darwin hablaba del más competente, el que sea capaz de adaptarse a las nuevas condiciones.

Asimismo, el Ministerio de Educación (MINEDU) asume como principio el enfoque curricular por competencias. Este punto es explicado mejor por (De Ketele, 2008; Jonnaert, 2001; Roegiers, 2010) citados por (Goñi, 2014), donde una competencia es un conocimiento especializado, compuesto y aplicado a las situaciones y contextos sociales diversos. Para estos autores, la competencia permite actuar en determinados contextos, es decir, una competencia es específica, por lo que no puede servir de manera unívoca en cualquier contexto. Al respecto Enkvist (1988) afirma que, dentro de la psicología, las fuentes del constructivismo se pueden encontrar en Piaget, Vigotsky y Ausubel, asimismo, en la filosofía de los idealistas alemanes. La mayoría de los constructivistas son filosóficamente idealistas como Kan. El constructivismo viene a ser un antropocentrismo porque la realidad pasa a verse como algo subjetivo. Con el constructivismo, la educación se aleja de la idea del estudioso que estudia para conocer la realidad.

La concepción cristiana por los principios que asume paradigmas educativos escolásticos e idealistas para ejercer el control de las masas, es muy evidente lo que afirma Gentili citado en (Ponce 1984) declarando que se debe confiar a la religión el control espiritual de la plebe, "estoy convencido -dice- de que, para formar un pueblo verdaderamente fuerte, es necesario que los ciudadanos tengan una concepción religiosa de la vida. Para conseguir ese resultado es necesario enseñar la religión a los niños". Es decir, un pueblo resignado y manso, una sociedad para quienes los únicos que tienen razón es la burguesía. A través de la religión cristiana se fomenta la resignación y la mansedumbre. Perú es una sociedad, donde se asume como algo natural los conceptos como "roba, pero hace obras", o como la frase de Abelardo Gutiérrez, Tongo "sufre peruano, sufre". Son síntomas de actitudes evasivas y resignación colectiva. Como predijo Honoré de Balzac, escritor francés del s. XIX, que la resignación es un suicidio cotidiano.

La religión cristiana desde los tiempos de Lutero, mediante su doctrina ha establecido, la fe como pretexto para justificar e imponer ciertas normas de conducta. Nietzsche,(1993) indica que, en todos los tiempos, por ejemplo, en el caso de Lutero, la fe no ha sido más que un manto, un pretexto, una cortina detrás del cual los instintos hacían de las suyas; una prudente ceguera para el imperio de determinados instintos. 
Las ideas cristianas como el constructivismo plantean el antropocentrismo, el culto al individuo, descartando la acción social. En este campo la religión incaica "tiene fines temporales más que fines espirituales. Se preocupaba del reino de la tierra antes que del reino del cielo. Constituía una disciplina social más que una disciplina individual" (Mariategui, 1928) . Los preceptos cristianos por medio de la salvación, el pecado, la fe mítica y el constructivismo, en el plano educativo, constituyen el individualismo egoísta, porque, "La religión tiene origen en un sentimiento egoísta" (Feurbach, 1948). El aprendiz con el modelo actual disipa la sensibilidad social, es que, elabora sus conocimientos en forma individual, aislado de la situación objetiva y bajo principios del colectivismo. Donde los saberes previos son los únicos medios para desarrollar el aprendizaje.

El constructivismo es un modelo determinista. Donde el estudiante pierde la capacidad, la razón crítica y la sensibilidad social. Por lo que se hace necesario generar el liderazgo social.

Es necesario promover las actitudes afectivas, cognitivas y conductuales. Es decir, el amor, el conocimiento crítico y la moral. Pero la religión anula estos elementos. Sobre el tema Cisneros (2000) señala en el Gran daño de la Religión, que ella "Las mutila despiadadamente. Les extirpa el pensamiento crítico. Les quita lo más preciado que posee el ser humano que es su libertad. Libertad de pensamiento propio". El modelo constructivista fomenta el oscurantismo, el escolasticismo donde soslaya la consciencia crítica y la libertad de pensamiento. $\mathrm{Y}$ es que la religión cristiana fomenta el temor mediante el castigo en el infierno (Apocalipsis) (Rt 1:2 Traductor Reina Valera 1960). Establece la auto-culpa, genera un pensamiento subjetivo. Con la religión el niño crece, físicamente atormentado, aterrorizado con la idea del castigo, "si te comportas mal, arderás en el infierno". El producto psicológico es un hombre sumiso, incapaz de luchar por sus derechos; asimismo genera la resignación, o sea, cumple con la demanda del sistema social. Entonces, si es obediente es un hombre calificado y aceptado. Si escapa a estos paradigmas ideológicos, es un anormal, en el extremo, es un terrorista. Así le sobre la razón, el sistema lo sataniza para que la sociedad confina en estereotipos sociales.

La religión cristiana junto al modelo constructivista aprehende el idealismo de Platón, como una concepción ideológica y filosófica. Las actitudes como la resignación, la dependencia, el temor y la apatía social, la ausencia de postura crítica; buscan anular el valor de la solidaridad para consolidar el individualismo. El psicoanalista Bruce ( 2011) indica que, "una masa crítica puede ser incómoda y embrutecerla es una necesidad". Las posturas religiosas son blandas, agnósticas, escépticas, también, totalitarias absolutistas según el momento.

En este estudio se ha determinado que la concepción cristiana influye en la actitud de liderazgo social de manera perniciosa, debido a que las manifestaciones conceptuales y las prácticas en relación a la fe, la salvación y el pecado generan pasividad, dependencia, el temor y la apatía social. En efecto, la religión tiene una relación de incidencia en la toma de actitudes negativas en el ámbito político social de los estudiantes. Lo cual evidentemente imposibilita el desarrollo del liderazgo social. Bajo esta misma línea Condori (2011) señala que a religión cristiana por contener preceptos e ideas celestiales como la fe divina, la salvación, el pecado y el castigo en el infierno generan conductas sumisas, indiferentes, dependientes, agresivas y timoratas. Asimismo, Pérez (2011) afirma que "requerimos sublevar el alma de los peruanos y sacudirnos de la sumisión, la apatía y la resignación que alimenta nuestro día a día".

Por su lado, Nietzsche (1997) considera que el uso de "los conceptos 'más allá', 'alma', juicio final, inmortalidad del alma; se trata de instrumentos de tortura, de sistemas de crueldades". Es Tan ruin que ha desarrollado el fanatismo, histeria colectiva y traumas psicopatológicos como se ha evidenciado en el estudio de (Condori, 2011). Estos conceptos cristianos impiden el desarrollo de la conciencia, la libertad de pensamiento, por ser dogmas ortodoxos y absolutistas. Convirtiéndose en escudos ideológicos, protectores del sistema vigente. El infortunio no es cosa de la religión más que la prédica por los de abajo reducido en las misas, donde se evoca, se pide por los necesitados, pero no existe una acción concreta a favor de ellos. La opresión del sistema se justifica, mediante "la democracia intervencionista", "sálvese 
quien pueda”, el más competente sobrevive a costa del más “tonto". Darwin (1859) y la selección natural evidencia este fenómeno, la capacidad de adaptarse a las circunstancias.

Cuando la religión responde a las necesidades del sistema, se promueve. Cuando la ideología y la conciencia rebasan sus límites; se prohíbe. Prohibido está el pensamiento científico, la filosofía y la ciencia política para la prole. Veto que se comprueba en los planes de estudio a nivel de Educación Básica y Superior. "Guárdate delante de él, y oye su voz; no le seas rebelde; porque él no perdonará vuestra rebelión, porque mi nombre está en él" (Éxodo, 23, 21), no hay duda de que la religión como doctrina es rígida, castiga, sanciona; no perdona. El pueblo es el rebaño con ceguera que debe seguir al pastor sin la posibilidad de crítica u opinión.

Por lo tanto, la concepción cristiana es totalitario, absolutista, dogmática e anticientífica lo que influye negativamente en la formación de liderazgo social. Esta ideología impregna el pragmatismo y el dogmatismo concordante con la política neoliberal donde se promueve el individualismo extremo contrario al espíritu sensible y solidario. Concepción impuesta en los planes curriculares del sistema educativo nacional, como es el caso de la Educación Religiosa (MINEDU, 2012). Con el propósito de formar hombres sin la capacidad de crítica, ni infundir la solidaridad y sometidos a la explotación.

El sistema imperante necesita difundir y reforzar el dogma para preservar los privilegios. Como en su estudio (Ponce, 2015) señala que, todo lo que se inculca no tiene ya como antes el propósito del bien común, sino en cuanto ese 'bien común' puede ser una premisa necesaria para mantener y reforzar a las clases dominantes. Para estas, la riqueza y el saber; para las otras, el trabajo y la sumisión.

En consecuencia, al determinar su objetivo, se concluye que la concepción cristiana influye en la actitud de liderazgo social de los estudiantes en general. Y este resultado será útil para las distintas instituciones educativas, pero sobre todo en las facultades de Ciencias de la Educación se establezca decisiones acertadas y elevar los niveles de liderazgo en los estudiantes, generando académicos y políticos con alta concepción científica, humanistas y con sensibilidad social. Asimismo, se demanda fortalecer la docencia universitaria con visión social, orientada hacia la formación de líderes sociales.

Sin embargo, los procesos productivos y el actuar organizacional están afectados por elementos económicos, pero además de ellos existen otros determinándolos, como son los sociales y los humanoculturales y que los efectos de las ideas religiosas en la formación de la mentalidad económica está implicada en el actuar de la organización (Anzola, 2003).

La teoría fundada será útil para remediar los problemas conceptuales y su praxis de los estudiantes, si los hubiere, en el tema de liderazgo. Consecuentemente, el presente estudio confiere el aporte metodológico ya que proveerá instrumentos validados para las futuras investigaciones respecto a temas de concepción y liderazgo.

Ha sido concluyente en la presente investigación, ya que el objetivo fue estudiar la influencia de la concepción cristiana y el hecho religioso con el método de las ciencias sociales, vinculado al credo cristiano.

\section{MATERIALES Y MÉTODO}

El método utilizado en la investigación es el descriptivo transversal. Aplicándose el diseño explicativo causal. Asimismo, se utilizó el diseño analítico y causal para caracterizar las variables de estudio en base a la observación (Charaja, 2009). Cuya población de investigación son los estudiantes del X semestre, V nivel de los programas de estudio de la Facultad de Ciencias de la Educación (FCEDUC UNA) ubicada en la ciudad de Puno - Perú.

\section{Técnica de recolección de datos}

La técnica de investigación que se ha empleado fue la encuesta, tanto en la variable independiente (concepción cristiana) como para la dependiente (liderazgo social). También se utilizó el análisis documental para determinar los conceptos de las dos concepciones del mundo. (Thurstone, 1928; Osgood, Suci, \& Tannenbaum, 1957) consideran que la actitud es una evaluación o una reacción afectiva, donde 
también se mide la actitud teniendo en cuenta si los sentimientos de un individuo hacían un objeto son favorables o desfavorables (Whittaker, 1979).

\section{Instrumento de recolección de datos}

Se aplicó guía de encuesta, dicho instrumento de recolección de datos se ha utilizado para medir ambas variables.

La Guía de Encuesta para la variable independiente consiste en un instrumento de 20 ítems con respuesta dicotómica SI-NO. Lo que significa que la respuesta "SI" equivale a 1 y la respuesta "NO", es igual a 0, ya que teniendo 20 ítems se tuvo un total de 20 puntos.

Por otro lado, el instrumento para la medición de la variable dependiente (liderazgo social) es el que consiste en un instrumento de 20 ítems, los mismos que siguieron el procedimiento planteado para la variable anterior y se ha empleado la misma escala de tabulación. En cuanto a la validez interna del instrumento de investigación se ha verificado la factibilidad a través de una prueba piloto utilizando el coeficiente de Alpha de Cronbach cuyo resultado se aproxima a ser altamente confiable (marcada) con una puntuación de 0,7445 puntos $(74,45 \%)$.

\section{RESULTADOS}

Tabla 1 Concepción cristiana en los estudiantes del X semestre de la Facultad de Ciencias de la Educación UNA Puno

\begin{tabular}{lcc}
\hline \multirow{2}{*}{ Categoría } & \multicolumn{2}{c}{ Concepción cristiana } \\
& $\mathrm{N}^{\mathbf{o}}$ & $\%$ \\
\hline Concepción cristiana muy alta & 46 & 41,8 \\
Concepción cristiana alta & 10 & 9,1 \\
Concepción cristiana regular & 7 & 6,4 \\
Concepción cristiana baja & 12 & 10,9 \\
Concepción cristiana nula & 35 & 31,8 \\
\hline Total & 110 & 100 \\
\hline
\end{tabular}

Fuente: Encuesta

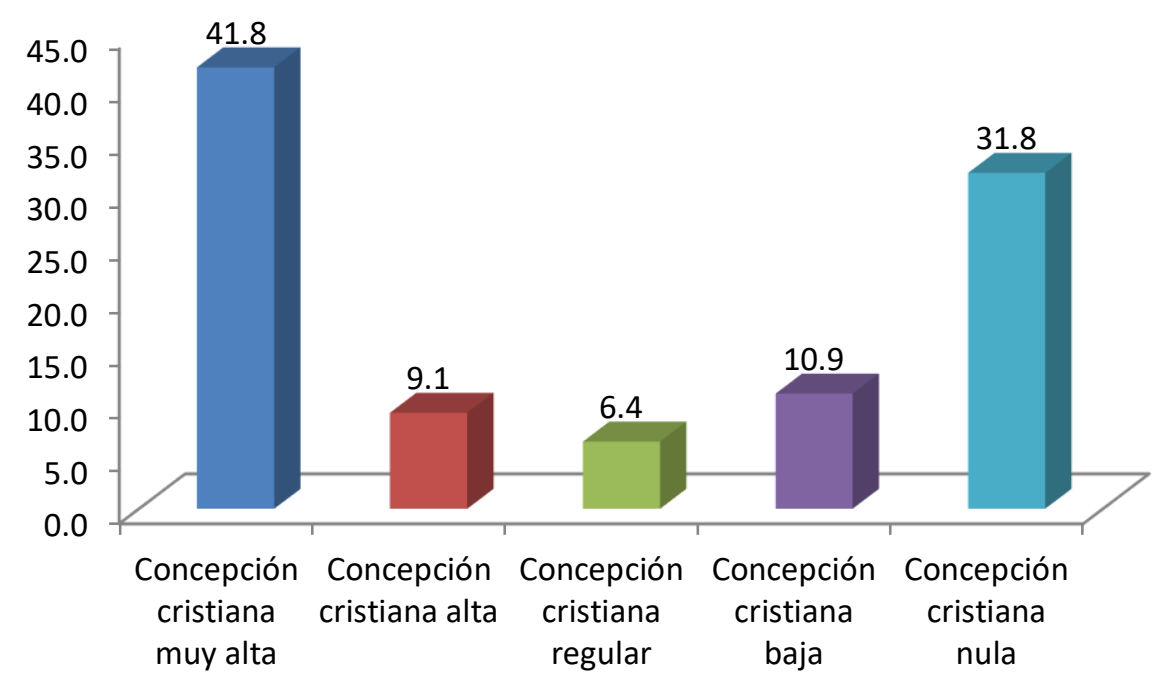


Figura 1. Concepción cristiana en los estudiantes del X semestre de la Facultad de Ciencias de la Educación UNA Puno

Según la tabla 1 y figura 1, en relación a la concepción cristiana de los estudiantes del X semestre de la Facultad de Ciencias de la Educación UNA Puno, se observa el predominio del 41,8\% de estudiantes, quienes presentan una concepción cristiana muy alta; en segundo lugar se ubica el 31,8\% de estudiantes quienes poseen una concepción cristiana nula.

De lo descrito se desprende la idea de un antagonismo entre dos posturas relacionadas a la concepción cristiana. Dos extremos irreconciliables que guían las ideas de los estudiantes. Las causas de este antagonismo son diversas, pero fundamentadas en las diferencias socio económicas de los estudiantes y de una visión influida por un sistema religioso y mediático que instruye a los estudiantes a que piensen de un modo contrario a su contexto.

Es importante destacar, también, el hecho de que ambas posturas predominantes llegan casi a la mitad de la muestra. Lo que significa que los estudiantes sí tienen definida su concepción cristiana afirmativa o negativa.

Por otro lado, los otros estudiantes se ubican en otras categorías calificadas como dubitativas, en el sentido de que sus afirmaciones no son directas, sino pertenecientes a escalas medianas.

Lo expresado, mediante un análisis comparativo con el estudio de (Sonera, 2009), difiere en cuanto a la muestra, debido a que la investigación que se hizo se dirige a estudiantes, mientras que Sonera, se dirige a docentes; no obstante ambos estudios se asemejan en lo que concierne a los resultados ya que, mediante los cuadros descritos se advierte el hecho de que los estudiantes en un 41,8\% están condicionados por la concepción cristiana, traducida como creencia religiosa cristiana; mientras que en el estudio de Sonera (2009) se arguye que el conocimiento de los informantes está condicionado (influenciado) por sus creencias religiosas.

En consecuencia, la concepción cristiana por desarrollar la compasión, la caridad y misericordia destruye la fe interna del hombre e impide la búsqueda de la libertad social. Este hecho aniquila la fuerza interna. El líder bajo este concepto se torna dependiente de las fuerzas externas. Y se desprende de la autoconfianza, inhibe sus propias capacidades, con ello evidentemente pierde la fuerza, la convicción y fe en su energía vital. El hombre con fe propia no necesita de poderes extraterrenales para resolver sus problemas. Porque el optimismo, la fuerza interna es la que vigoriza y es el motor del desarrollo humano. El optimismo es el motor interno de la persona, es decir, su propia fe que desarrolla convicción.

\section{Actitud de Liderazgo Social}

Tabla 2 Actitud Liderazgo Social en los estudiantes del X semestre de la Facultad de Ciencias de la Educación UNA Puno

\begin{tabular}{llc}
\hline \multirow{2}{*}{ Categoría } & \multicolumn{2}{l}{ Actitud de Liderazgo Social } \\
& $\mathbf{N}^{\mathbf{0}}$ & $\mathbf{\%}$ \\
\hline Liderazgo social muy bueno & 22 & 20,0 \\
Liderazgo social bueno & 15 & 13,6 \\
Liderazgo social regular & 11 & 10,0 \\
Liderazgo social deficiente & 12 & 10,9 \\
Liderazgo social muy deficiente & 50 & 45,5 \\
\hline Total & $\mathbf{1 1 0}$ & 100 \\
\hline
\end{tabular}

Fuente: Encuesta 


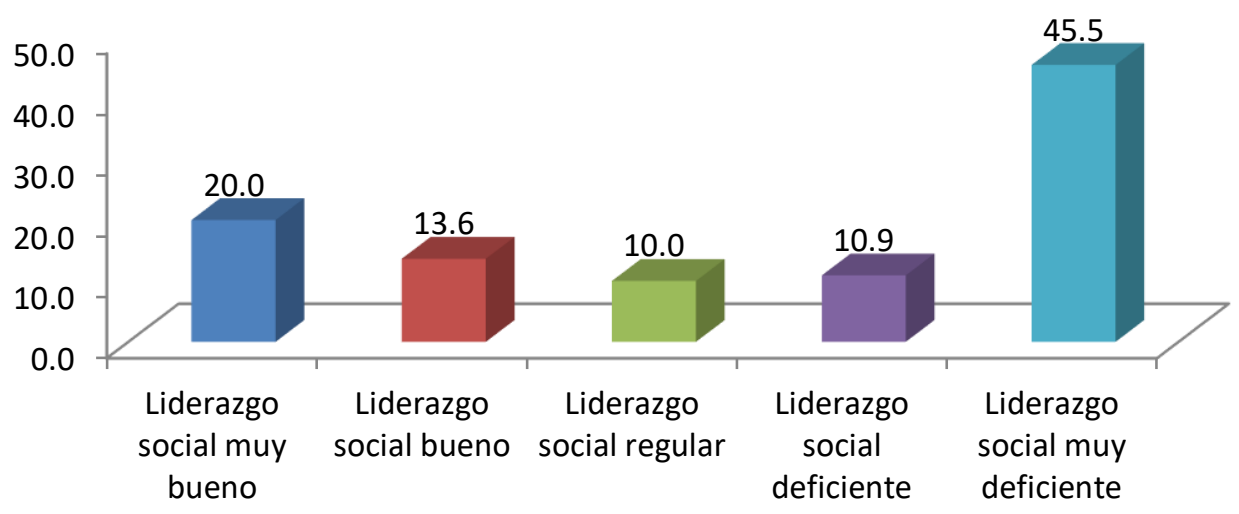

Figura 2. Actitud Liderazgo Social en los estudiantes del X semestre de la Facultad de Ciencias de la Educación UNA Puno

En tabla 2 y figura 2, en relación a la actitud de liderazgo social en los estudiantes del X semestre de la Facultad de Ciencias de la Educación UNA Puno, se observa el predominio del 45,5\% de estudiantes, quienes presentan un liderazgo social muy deficiente; en segundo lugar, se ubica el $20 \%$ de estudiantes, quienes poseen un liderazgo social muy bueno.

Estos resultados permiten afirmar que la tasa de liderazgo en la universidad ha ido decreciendo a lo largo de los años, debido a factores socioeconómicos. Una de las evidencias de lo manifestado es la deficiente participación de estudiantes en situaciones sociales de movilización y participación en actividades socio político, académico y científico.

Por ello, la Universidad no se encuentra en expectantes lugares en las evaluaciones internacionales en el plano político, social y académico. Tampoco se visualiza líderes con visión social.

Mediante un análisis comparativo con otros estudios, se encontró que según Blanco (2011) las fuerzas conservadoras y el poder impiden el desarrollo de actitudes críticas y de liderazgo constructivo. Ello se asemeja al resultado de este ítem, cuando se observa que un 45,5\% presenta un liderazgo deficiente.

Por su parte, Marín ( 1979) indica que los componentes para generar un liderazgo social son los procesos cognitivos que comprenden las sensaciones, percepciones, memoria, pensamiento, la imaginación como procesos mentales, llamada como actitud cognitiva, La actitud afectiva involucra la emoción, el sentimiento, la pasión y los estados de ánimo. Los procesos conativos, es decir lo conductual, comprenden las motivaciones, los intereses, las necesidades y la voluntad.

En el presente estudio se requieren ejecutar las propuestas señaladas, debido al alto índice de deficiencia de liderazgo, con la finalidad de eliminar la pasividad, sumisión y mansedumbre, cualidades de los dirigentes (Lora, 1989). Como se ha demostrado, los principios cristianos buscan anular el valor de la solidaridad consolidando el individualismo. El psicoanalista Bruce (2011) dice: "Una masa crítica puede ser muy incómoda. Y embrutecerla es una necesidad”. Para ello se utiliza la disposición hacía lo misterioso y lo sobrenatural que todo ser humano posee. El problema está cuando se cae en el fenómeno de la desrealización. Es decir, tomamos como verosímiles sucesos que a todas luces son fabricaciones grotescas. Cortinas de humo como las vírgenes que lloran o los pishtacos, cuya intencionalidad es mantener el control político y hegemónico...son apología a la idiotez. Y es falso que tengas la opción de leer o ver otros contenidos cuando la televisión cerrada la prensa están plagadas de lo mismo. 


\section{DISCUSIÓN}

Esta investigación plantea como propósito estudiar la influencia y el hecho religioso con el método de las ciencias sociales, vinculando a un credo específico y concreto, el cristianismo. La expresión de la conducta religiosa, la religiosidad, se puede encontrar en las diversas dimensiones de la vida personal y social. Se puede hablar de religión como un modo de sentimiento, un modo de pensar, un modo de relacionarse.

El grupo estudiado ha sido seleccionado considerando aspectos comunes en cuanto a ambas variables. El grado de adecuación para el estudio fue óptimo debido a que los instrumentos fueron validados.

De los resultados obtenidos, se infiere que la concepción cristiana influye en la actitud de liderazgo social de los estudiantes del X semestre de la Facultad de Ciencias de la Educación FCEDUC UNA Puno - 2014. Mediante un análisis comparativo con otro estudio sobre aspectos actitudinales vinculados a la religión, se observa que según Weber (1905) las "ideas puritanas" han influido en el desarrollo del capitalismo; es decir el movimiento religioso influyó sobre el desarrollo de la cultura material en virtud de similitudes de opción. En ese plano, determina que la religión fue uno de los aspectos más importantes que influyó en el desarrollo de las culturas occidental y oriental. Semejantes resultados y conclusiones destacan también en la presente investigación.

Por otra parte, el estudio de Sonera (2009) permite comprender y explicar cómo operan las creencias religiosas en el desarrollo de las versiones que sostienen los maestros con respecto a la Teoría de Evolución Biológica (TEB). El modelo sintetiza el hecho de que la postura o versión hegemónica que asume el docente en relación con esta explicación científica se vincula con el valor que otorgan a las creencias religiosas que profesan. En este sentido el modelo postula que el conocimiento de los informantes está condicionado por sus creencias religiosas. Similares coincidencias se encuentran también en la presente investigación.

Condori (2011)describe tipos de conducta que genera la influencia de la Religión Cristiana en los niños y niñas de la Institución Educativa Primaria Cuturapi, para afirmar que los tipos de conducta que presentan los niños y niñas, como producto de la influencia de la Religión Cristiana, son sumisas, egoístas e individualistas. Un total de $89 \%$ de los niños estudiados no son críticos frente a la enseñanza de los preceptos cristianos.

Por otro lado, los resultados de la presente investigación señalan que existe influencia significativa de la primera sobre la segunda variable, considerando las hipótesis de investigación; así entre las variables concepción cristiana y actitud de liderazgo social se destaca mediante Chi cuadrado, es contundente: $|164,6|>|8,0|$; es decir: $\left|X^{2}{ }_{o}\right|>\left|X^{2}{ }_{c}\right|$, resultados totalmente contrarias de (Beltrán, 2013), en la que indica que, los nuevos movimientos religiosos predican valores como la dedicación al trabajo, la honestidad, la responsabilidad y la disciplina. Además, fomentan habilidades y competencias propias del liderazgo, como el trabajo en equipo, la iniciativa y la oratoria, competencias que son útiles también en los ámbitos seculares, inclusive hasta la constitución de espacios para la creación y reproducción de capital social.

En cuanto a las líneas de investigación que surgen de los resultados, se propone como campos nuevos de estudio: la labor pedagógica del docente se debe desvincular de las creencias religiosas arbitrarias para con los estudiantes según en el marco de un nuevo modelo de gestión acorde al siglo XXI. Es conveniente generar la educación laica en los centros de formación superior. También estilos de liderazgo social diseñados desde el seno del pueblo y no de la religión, principalmente la cristiana. Porque, es una realidad que gran porcentaje de creyentes practicantes y participantes de misas, cultos y celebraciones religiosas crece en los estratos socioeconómicos más bajos, en las zonas urbanas excluidas, entre las comunidades indígenas y, en general, donde el estado es débil (Beltrán, 2013). Incluso, se debe entender como un patrón conductual que ha evolucionado junto con la capacidad humana de trabajar en equipo y coordinar las acciones individuales y especializadas en patrones de comportamientos más efectivos y complejos 
(Ramírez, 2013). En este escenario un líder debe poseer valores inmanentes que permitan guiar al grupo hacia un derrotero final y beneficioso(Terrazas, 2015), y debe ser un instrumento de transformación espiritual para los demás(Terrazas, 2015).

Además, el relativo éxito alcanzado por el partido es producto, entre otras cosas, del descrédito de los partidos tradicionales, la propuesta del partido como renovador de los vicios políticos y la expansión de sus iglesias en el territorio nacional (Valderrama, 2011), y en vez de "pensar en rescatar al mundo como tal", es "pensar en rescatar los corazones para rescatar al mundo". Se trata de intentar restablecer el respeto y temor hacia Dios (Terrazas, 2015). En contraste con otros tipos de liderazgos, el cristiano tiene que ver con la voluntad del ser humano en participar en este tipo de actividades que le brindan el conocimiento del bien y el mal; y le dan la oportunidad de ayudar al prójimo (Yasmari, 2018).

La función de la religión como elemento que la refuerza y que facilita la canalización de la energía de las personas en la dirección del trabajo (Anzola, 2003), es que la forma en que cómo se conduce al equipo de trabajo para el logro de los objetivos organizacionales, es lo que prima, tiene un efecto cascada que además impacta en su sentir y en sus comportamientos auténticos (Pautt, 2011). Por último, la investigación tiene una validez externa alta, debido a que los hallazgos realizados pueden aplicarse a otras poblaciones o muestras, es decir pueden extenderse a otras instituciones superiores universitarias con similares características.

\section{CONCLUSIONES}

La concepción cristiana influye en la actitud de liderazgo social de manera negativa en los estudiantes del X semestre de la Facultad de Ciencias de la Educación FCEDUC UNA Puno - 2014, debido a que las manifestaciones conceptuales y prácticas en relación a la fe, salvación y pecado buscan la pasividad y dependencia, consolidando actitudes negativas en el liderazgo que al mismo tiempo sea útil a la sociedad. Esta aseveración queda confirmada mediante el diseño estadístico Chi cuadrada, que fue contundente: $|164,6|>|8,0|$; es decir: $\left|X^{2}{ }_{o}\right|>\left|X^{2}{ }_{c}\right|$.

La práctica de la fe cristiana influye en la actitud de liderazgo afectivo de los estudiantes del $\mathrm{X}$ Semestre de la FCEDUC UNA - Puno, de manera negativa ya que dicha práctica genera indiferencia ante diversas situaciones políticas y sociales. Porque Dios no permite el desarrollo de la confianza, la fe interna; sino, lo maldice. Lo cual, evidentemente aniquila la fuerza interna, la convicción y fe en su energía vital. Profundiza la compasión, la caridad y la misericordia, en vez de generar la emoción, el sentimiento, pasión y el amor por la sociedad. Lo cual se refleja en los actuales líderes sin actitud de servicio al pueblo. Lo manifestado se confirma mediante el diseño Chi cuadrada: $|141,4|>|8,0|$; es decir: $\left|X^{2}{ }_{o}\right|>\left|X^{2}{ }_{c}\right|$.

La salvación cristiana incide en la actitud de liderazgo cognitivo en forma negativa en los estudiantes del X semestre de la FCEDUC UNA - Puno, debido a que la concepción cristiana sobre la salvación no permite el desarrollo de ideas revolucionarias ni muestra originalidad. Ssegún este precepto, el hombre no puede asumir la salvación social, porque esa facultad sólo pertenece a Dios. Por el contrario, buscan paradigmas individualistas, evitando el trabajo colectivo. Ello se ve contrastado con el diseño estadístico Chi cuadrado: $|157,6|>|5,2|$; es decir, $\left|X^{2}{ }_{o}\right|>\left|X^{2}{ }_{c}\right|$ el pecado repercute en forma negativa en la actitud de liderazgo conductual de los estudiantes del X semestre de la FCEDUC UNA - Puno, debido a que la creencia en el pecado infunde temor y causa inseguridad en la conducta. Porque este principio considera que todo hombre es pecaminoso tanto espiritual y corporal, cometida contra la ley de Dios. Y, el pecado da lugar a la muerte. Lo que menoscaba la voluntad, el deseo y la motivación social. Esta aseveración queda confirmada mediante el diseño Chi cuadrada $|171,4|>|8,0|$; es decir: $\left|X^{2}{ }_{o}\right|>\left|X^{2}{ }_{c}\right|$. De tal manera que, la religiosidad, sus instituciones o nuevas manifestaciones asumen una mayor visibilidad, ocupando espacios públicos y mediáticos; sus manifestaciones se pluralizan y se diversifican cada vez más ( Pérez, 2009). 


\section{REFERENCIAS BIBLIOGRÁFICAS}

Anzola, O. (2003). Influencia de lo religioso en la vida y la cultura corporativa. la sociedad de los testigos de Jehová: hogar Betel, Facatativá. Cuadernos de Administración, 16(26), 129-160. Recuperado de https://www.redalyc.org/pdf/205/20502607.pdf

Beltrán, w. (2013). Theologica Xaveriana. Theologica Xaveriana, 63 (175), 57-85. Recuperado de http://www.redalyc.org/articulo.oa?id=191028791003

Blanco, C. (2011). Actitudes críticas y fuerzas conservadoras. Lima Perú.

Bruce, J. (2011). Televisión Basura. (Hildebrandt, Ed.).

Charaja, F. (2009). MAPIC, En la Metodología de la Investigación. (N. Mundo, Ed.). Puno.

Cisneros, C. (2000). El gran daño de la religión. Revista de Opinión, 20-21.

Condori, Y. (2011). Influencia de la Religión Cristiana en la Conducta de los niños y niñas de I.E.P La Reforma de Cuturapi. Puno: Universidad Nacional del Altiplano. Universidad Nacional del Altiplano.

Darwin, C. (1859). El origen de las especies. (The Origin of Species o On the Origin of Species by Means of Natural Selection, or the Preservation of Favoured Races in the Struggle for Life.

De Ketele, J. (2008). Enfoque socio-histórico de las competencias en la enseñanza. Profesorado. Revista de currículum y Formación del Profesorado. Recuperado de http://www.ugr.es/local/recfpro/rev123ART1.pdf

Enkvist, I. (1988). Representaciones sociales y psicológicas de la religión. (Limusa., Ed.). México.

Feurbach, L. A. (1948). Esencia de la Religión. (Editorial Rosario S.A., Ed.). Rosario Argentina.

Goñi, J. (2014). Hacia un currículum guiado por las competencias. Pamplona: Universidad Pública de Navarra. (U. P. de Navarra, Ed.). Pamplona.

Jonnaert, P. (2001). Competencias y socioconstructivismo. Nuevas referencias para los programas de estudios. Texto de apoyo a la Segunda Conferencia Anual de Inspectores de la Enseñanza Media, Bobo Dioulasso, Burkina Faso, 18-22 de diciembre. (Bobo Dioul).

Lora, J. (1989). El maestro Encinas. (Perú, Ed.). Juliaca.

Mariategui, J. C. (1928). Siete Ensayos de Interpretación de la Realidad Peruana. Lima - Perú: Editora Amauta S.A. (Amauta S.A, Ed.). Lima Perú.

Marín, G. (1979). Componentes de la Estructura de Actitudes. (Trilles, Ed.). México.

MINEDU. (2012). Manual del usuario para la aplicación del SIAGIE 3.0. Lima Perú.

Nietzsche, F. (1993). El Anticristo. (Colicheuque, Ed.). México.

Osgood, C., Suci, G., \& Tannenbaum, P. (1957). The Measurement of meaning. Illinois: University of Illinois Press.

Pautt, G. (2011). Liderazgo y dirección: dos conceptos distintos con resultados diferentes. Revista Facultad de Ciencias Economicas, XIX (1), 213-228. Recuperado de https://www.redalyc.org/pdf/909/90922732013.pdf

Pérez, N. (2009). El fenómeno religioso y su importancia para el análisis de la realidad sociopolítica cotidiana. El Cotidiano, 156(156), 345-356. Recuperado de http://www.redalyc.org/pdf/325/32512743022.pdf

Pérez, W. (2011). La indiferencia del peruano. Recuperado de http://wperezruiz.blogspot.pe/2011/09/laindiferencia-del-peruano.html

Ponce, A. (2015). Educación y lucha de clases y otros escritos. (UNIPE, Ed.). Editorial Universitaria.

Quiroz, H. (1998). Corrientes Pedagógicas Contemporáneas. (Redes, Ed.). Lima.

Ramírez, G. (2013). Liderazgo organizacional. Un desafío permanente. Universidad \& Empresa, 15(25), 5-11. Recuperado de https://www.redalyc.org/pdf/1872/187229746001.pdf

Roegiers, X. (2010). Una pedagogía de la integración. Competencias e integración de los conocimientos 
en la enseñanza. (F. de C. Económica., Ed.). México.

Sonera, J. (2009). Influencia de las Creencias Religiosas en los Docentes de Ciencia sobre la Teoría de la Evolución Biológica y su Didáctica. CREB.

Terrazas, R. (2015). Liderazgo Tridimensional. Perspectivas, 35, 7-28. Recuperado de http://www.redalyc.org/pdf/4259/425941232002.pdf

Thurstone, L. (1928). Attitudes can be measured. American Journal of Sociology, 33, 529-554.

Valderrama, L. A. (2011). El pentecostalismo en Colombia. Prácticas religiosas, liderazgo y participación política. Franciscanum. Revista de las ciencias del espíritu, LIII(155), 199-208. Recuperado de http://www.redalyc.org/articulo.oa?id=343529075009

Weber, M. (1905). La Ética Protestante y el Espíritu del Capitalismo. (Limusa, Ed.). México.

Whittaker, J. (1979). La Psicoloclía Social en el Mundo de Hoy. (Trillas, Ed.). México.

Yasmari B. (2018). Liderazgo Cristiano o Religioso | Yasmaribello. Recuperado 14 de mayo de 2019, de https://yasmaribello.com/liderazgo-cristiano/ 